\title{
Applications of Composite Numerical Integrations Using Gauss-Radau and Gauss-Lobatto Quadrature Rules
}

\author{
M. A. Hossain ${ }^{1}$ and M. S. Islam ${ }^{2}$ \\ ${ }^{1}$ Department of Mathematics, Jagannath University, Dhaka-1100, Bangladesh \\ ${ }^{2}$ Department of Mathematics, University of Dhaka, Dhaka-1000, Bangladesh \\ Received 25 May 2010, accepted in revised form 18 July 2010
}

\begin{abstract}
In this paper, numerical integrals over an arbitrary triangular region are evaluated exploiting finite element method. The physical region is transformed into a standard triangular finite element using the basis functions in local space. Then the standard triangle is discretized into $4 \times n^{2}$ right isosceles triangles, in which each of these triangles having area $1 / 2 n^{2}$, and thus composite numerical integration is employed. In addition, the affine transformation over each discretized triangle and the use of linearity property of integrals are applied. Finally, each isosceles triangle is transformed into a 2-sqare finite element to generate new $n^{2}$ extended sampling points and corresponding weight coefficients, using $n$ point's conventional Gauss-Radau and Gauss-Lobatto quadratures, which are applied again to evaluate the double integral. The performance is depicted by means of numerical examples.
\end{abstract}

Keywords: Double integral; Numerical Integration; Quadrilateral and Triangular Finite Element; Gauss-Radau and Gauss-Lobatto quadratures.

(C) 2010 JSR Publications. ISSN: 2070-0237 (Print); 2070-0245 (Online). All rights reserved.

DOI: 10.3329/jsr.v2i3.5123 J. Sci. Res. 2 (3), 465-467 (2010)

\section{Introduction}

Numerical simulation in engineering science and in applied mathematics has become a powerful tool to model the physical phenomena, particularly when analytical solutions are not available and/or are very difficult to obtain. The integrals arising in practical problems are not always simple and the quadrature scheme cannot evaluate with desired accuracy, the composite numerical integration is dealt then to obtain high accuracy.

From the literature review we observe that numerical integrations over triangular regions were first introduced by Hammer and co-workers [1-3]. In the working of finite

\footnotetext{
${ }^{2}$ Corresponding author: mdshafiqul@yahoo.com
} 
element method, the triangular elements are widely used in the area of numerical integration schemes [4]. The work of Hammer et al. [1] has been further developed by Cowper [5], and thus he provided a table of Gaussian quadrature formulae for symmetrically placed integration points. Lyness and Jespersen [6] derived symmetric quadrature rules and provided integration formulas with a precision of upto degree eleven by formulating the problem in terms of polar coordinates. Lether [7] and Hillion [8] derived formulas for triangles as product of one-dimensional Gauss quadrature rule. Lannoy [9] discussed the symmetric four-point integration rule. Lague and Baldur [10] included a technique for numerical integration over the surface of a triangle. Laursen and Gellert [11] also discussed elaborately symmetric integration formulae of precision up to degree ten. In ref. [12], double integrals over a standard triangular region are evaluated. In ref. [13], Gauss-Radau and Gauss-Lobatto quadrature rules are presented to evaluate the rational integrals of the element matrix for a general quadrilateral. In [14], double integrals over an arbitrary quadrilateral are evaluated. In [14], the physical region is transformed into a standard quadrilateral finite element using the basis functions in local space. Then the standard quadrilateral is subdivided into two triangles, and each triangle is further discretized into $4 \times n^{2}$ right isosceles triangles, with area $1 / 2 n^{2}$, and thus composite numerical integration is employed.

In this paper an arbitrary triangular region is transformed into a standard triangular finite element using the basis functions in local space. Then the standard triangle is discretized into $4 \times n^{2}$ right isosceles triangles. Moreover, each isosceles triangle is transformed into a 2-sqare finite element to compute new $n^{2}$ extended Gauss Radau and Gauss Lobatto points and corresponding weight coefficients, using $n$ point's, Gauss Radau and Gauss Lobatto quadratures are used instead of Gauss Lagendre quadrature [14], which are applied again to evaluate the double integral. The subsequent formulations are developed by Mathematica. The performance of the present formulation is excellent.

\section{Numerical Integration}

The most common numerical integration [13] is

$$
\int_{a}^{b} w(x) f(x) d x=\sum_{k=1}^{n} w_{k} f\left(x_{k}\right)+E[f]
$$

known as the Gaussian quadrature, where $x_{k}, w_{k}(k=1,2, \ldots . ., n)$ and $E[f]$ are called nodes (abscissas), weights, and error approximation, respectively.

Assuming the exactness (i.e. $E[f]=0$ ) and for our convenient ( by change of variable), and set $w(x)=1$, the equation (1) can be written as,

$$
\int_{1}^{-1} w(x) f(x) d x=\sum_{k=1}^{m} w_{k} f\left(x_{k}\right)+\sum_{k=m+1}^{n-m} w_{k} f\left(x_{k}\right) \quad m \leq n
$$


If we put $m=0$, then the concluded numerical integration rule is called GaussLegendre. If we put $m=1, x_{1}=-1$ (or 1 ) and then the concluded numerical integration rule is called Gauss-Radau [13]. If we put $m=2, x_{1}=-1$ and $x_{\mathrm{n}}=1$ and then the concluded numerical integration rule is called Gauss-Lobatto [13].

\section{Formulation of Integrals Over an Arbitrary Triangular Region}

The integral of an arbitrary function, $f(x, y)$ over an arbitrary triangular region $A T$ is given by

$$
I=\iint_{A T} f(x, y) d y d x=\iint_{A T} f(x, y) d x d y
$$

The integral $I$ of Eq. (3) is then transformed into an integral over the region of the standard triangle $S T=\{(u, v):-1 \leq v \leq 1,-1 \leq u \leq-v\}$ by the liner triangular finite element basis functions $L_{i}(u, v)$, shown in Fig. 1:

$$
L_{1}(u, v)=-\frac{1}{2}(u+v), L_{2}(u, v)=\frac{1}{2}(1+u) \text { and } L_{3}(u, v)=\frac{1}{2}(1+v) .
$$

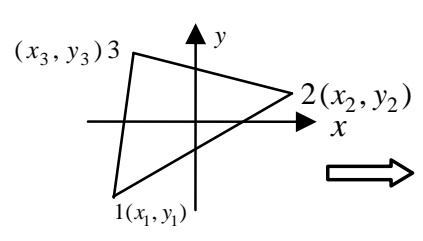

(a) Arbitrary triangle $A T$

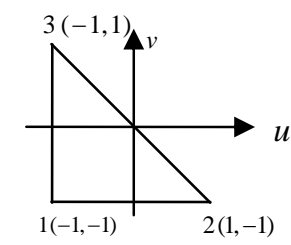

(b) Standard triangle $S T$

Fig. 1. Transformation of arbitrary triangle $A T$ into equivalent standard triangle ST.

The coordinates are changed by assuming that

$$
x=\sum_{i=1}^{3} x_{i} L_{i} \text { and } y=\sum_{i=1}^{3} y_{i} L_{i}
$$

$$
\text { and the corresponding Jacobian, } J_{1}=\frac{\partial(x, y)}{\partial(u, v)}=\frac{\partial x}{\partial u} \frac{\partial y}{\partial v}-\frac{\partial x}{\partial v} \frac{\partial y}{\partial u}
$$

Therefore using Eqs. (4) and (3) to obtain,

$$
I=\iint_{A T} f(x, y) d y d x=\iint_{S T} f(u, v)\left|J_{1}\right| d u d v
$$

The integral $I$ of Eq. (5) can be further transformed into an integral over the standard 2-square, $\{(\xi, \eta):-1 \leq \xi, \eta \leq 1\}$ using standard quadrilateral basis functions $Q_{i}(\xi, \eta)$, as shown in Fig. 2: 


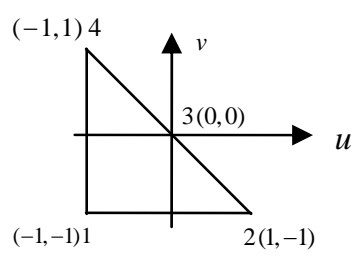

(a) Standard triangle $S T$

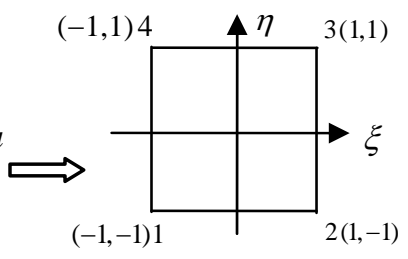

(b) Standard square element

Fig. 2. Transformation of standard triangle ST into 2-square.

Assume that

$$
\begin{aligned}
& u=\sum_{i=1}^{4} u_{i} Q_{i}=\frac{1}{4}(-1+3 \xi-\eta(1+\xi))=u(\xi, \eta) \\
& v=\sum_{i=1}^{4} v_{i} Q_{i}=\frac{1}{4}(-1+3 \eta-\xi(1+\eta))=v(\xi, \eta)
\end{aligned}
$$

and $J_{2}=\frac{\partial u}{\partial \xi} \frac{\partial v}{\partial \eta}-\frac{\partial u}{\partial \eta} \frac{\partial v}{\partial \xi}=\frac{1}{4}(2-\eta-\xi)$

Note that $J_{1}$ depends on the vertices of the given arbitrary triangular region, but $J_{2}$ is fixed.

Let $F(u, v)=f(u, v)\left|J_{1}\right|$ and using (6), Equ. (5) becomes

$$
\begin{aligned}
I & =\int_{-1}^{1} \int_{-1}^{-u} F(u, v) d v d u=\int_{-1}^{1} \int_{-1}^{1} F(u(\xi, \eta), v(\xi, \eta))\left|J_{2}\right| d \xi d \eta \\
& =\int_{-1}^{1} \int_{-1}^{1} F\left(\frac{1}{4}(-1+3 \xi-\eta(1+\xi)), \frac{1}{4}(-1+3 \eta-\xi(1+\eta))\right) \frac{1}{4}(2-\eta-\xi) d \xi d \eta
\end{aligned}
$$

Now Eq. (5) represents an integral over the standard 2-square region:

$\{(\xi, \eta):-1 \leq \xi, \eta \leq 1\}$. Hence using Gaussian quadrature rule for the integral $I$ of Eq. (7), we have

$$
I=\sum_{i=1}^{s} \sum_{j=1}^{s} \frac{1}{4}\left(2-\eta_{j}-\xi_{i}\right) w_{i} w_{j} F\left(\frac{1}{4}\left(-1+3 \xi_{i}-\eta_{j}\left(1+\xi_{i}\right)\right), \frac{1}{4}\left(-1+3 \eta_{j}-\xi_{i}\left(1+\eta_{j}\right)\right)\right)
$$

where $\left(\xi_{i}, \eta_{j}\right)$ are Gauss- Radau points and Gauss- Lobatto points in the $\xi, \eta$ directions of order $s$ and $w_{i}, w_{j}$ are the corresponding weight coefficients. We can write Eq. (8) as:

$$
I=\sum_{k=1}^{N=s \times s} c_{k} F\left(x_{k}, y_{k}\right)
$$

where $c_{k}, x_{k}$ and $y_{k}$ can be written in the form: 


$$
\begin{aligned}
c_{k} & =\frac{1}{4}\left(2-\eta_{j}-\xi_{i}\right) w_{i} w_{j} \\
x_{k} & =\frac{1}{4}\left(-1+3 \xi_{i}-\eta_{j}\left(1+\xi_{i}\right)\right),(k=1,2, \ldots \ldots \ldots \ldots, N),(i, j=1,2, \ldots \ldots \ldots \ldots, s) \\
y_{k} & =\frac{1}{4}\left(-1+3 \eta_{j}-\xi_{i}\left(1+\eta_{j}\right)\right)
\end{aligned}
$$

The weighting coefficients $c_{k}$ and sampling points $\left(x_{k}, y_{k}\right)$ of various order can be now easily computed from Eq. (10). In Table 1 and Table 2, values of, $c_{k}, x_{k}$ and $y_{k}$ for $s$ $=3,4,5,6$ are calculated by using Gauss- Radau points and Gauss- Lobatto points respectively. In both table sampling points are calculated by using Mathematica.

Table 1. Output of $c_{k}, x_{k}$ and $y_{k}$ of Eq. (10) using Gauss-Radau points.

\begin{tabular}{cccc}
\hline$k$ & \multicolumn{1}{c}{$c_{k}$} & $x_{k}$ & $y_{k}$ \\
\hline \multicolumn{4}{c}{ Order of Gauss-Radau Quadrature rule, $\mathrm{s}=3$} \\
\hline 1 & 0.096614387479324 & -1.000000000000000 & 0.689897948556636 \\
2 & 0.308641975308642 & -0.589897948556636 & 0.389897948556636 \\
3 & 0.087870061825481 & -0.024040820577346 & -0.024040820577346 \\
4 & 0.187336229804627 & -1.000000000000000 & -0.289897948556636 \\
5 & 0.677562036939952 & -0.415959179422655 & -0.415959179422655 \\
6 & 0.308641975308642 & 0.389897948556636 & -0.589897948556636 \\
7 & 0.049382716049383 & -1.000000000000000 & -1.000000000000000 \\
8 & 0.187336229804627 & -0.289897948556636 & -1.000000000000000 \\
9 & 0.096614387479324 & 0.689897948556636 & -1.000000000000000 \\
\hline & Order of Gauss-Radau Quadrature rule, s $=4$ & \\
\hline 1 & 0.029999063576822 & -1.000000000000000 & 0.822824080974592 \\
2 & 0.127051925813598 & -0.768848646756411 & 0.629294357739875 \\
3 & 0.085249046429649 & -0.357152738936900 & 0.284605070919161 \\
4 & 0.017222770232317 & -0.007847826570624 & -0.007847826570625 \\
5 & 0.068393228915845 & -1.000000000000000 & 0.181066271118531 \\
6 & 0.305638842571568 & -0.700713047374403 & 0.055672147265822 \\
7 & 0.246817075893281 & -0.167663113074927 & -0.167663113074927 \\
8 & 0.085249046429649 & 0.284605070919161 & -0.357152738936900 \\
9 & 0.073482707507333 & -1.000000000000000 & -0.575318923521694 \\
10 & 0.340705524244772 & -0.620407427701387 & -0.620407427701387 \\
11 & 0.305638842571568 & 0.055672147265822 & -0.700713047374403 \\
12 & 0.127051925813598 & 0.629294357739875 & -0.768848646756411 \\
13 & 0.015625000000000 & -1.000000000000000 & -1.000000000000000 \\
14 & 0.073482707507333 & -0.575318923521694 & -1.000000000000000 \\
15 & 0.068393228915845 & 0.181066271118531 & -1.0000000000000000 \\
16 & 0.029999063576822 & 0.822824080974592 & -1.0000000000000000 \\
\hline & & &
\end{tabular}




\begin{tabular}{cccc}
\hline \multicolumn{4}{c}{ Order of Gauss-Radau Quadrature rule, $\mathrm{s}=5$} \\
\hline 1 & 0.012153616652077 & 1.000000000000000 & 0.885791607770965 \\
2 & 0.058825725455510 & 0.852259260953794 & 0.754012618129610 \\
3 & 0.057423793701357 & 0.559811698754950 & 0.493160773753848 \\
4 & 0.027006093644855 & 0.235547715267332 & 0.203929919779881 \\
5 & 0.004717626056903 & 0.003260889213885 & 0.003260889213885 \\
6 & 0.028739796983092 & 1.000000000000000 & 0.446313972723752 \\
7 & 0.142753113111973 & 0.821548593625632 & 0.345245650410559 \\
8 & 0.150978996962694 & 0.468310352758179 & 0.145184484703406 \\
9 & 0.087660908725231 & 0.076642054200238 & 0.076642054200238 \\
10 & 0.027006093644855 & 0.203929919779881 & 0.235547715267332 \\
11 & 0.039504439867460 & 1.000000000000000 & 0.167180864737833 \\
12 & 0.200893758605095 & 0.778677615996012 & 0.225378209421406 \\
13 & 0.226983484574152 & 0.340577792752539 & 0.340577792752539 \\
14 & 0.150978996962694 & 0.145184484703406 & 0.468310352758179 \\
15 & 0.057423793701357 & 0.493160773753848 & 0.559811698754950 \\
16 & 0.033202146497371 & 1.000000000000000 & 0.720480271312439 \\
17 & 0.171275017680751 & 0.740013090993831 & 0.740013090993831 \\
18 & 0.200893758605095 & 0.225378209421406 & 0.778677615996012 \\
19 & 0.142753113111973 & 0.345245650410559 & 0.821548593625632 \\
20 & 0.058825725455510 & 0.754012618129610 & 0.852259260953794 \\
21 & 0.006400000000000 & 1.000000000000000 & 1.000000000000000 \\
22 & 0.033202146497371 & 0.720480271312439 & 1.000000000000000 \\
23 & 0.039504439867460 & 0.167180864737833 & 1.000000000000000 \\
24 & 0.028739796983092 & 0.446313972723752 & 1.000000000000000 \\
25 & 0.012153616652077 & 0.885791607770965 & 1.000000000000000 \\
\hline
\end{tabular}

Table 2. Output of $c_{k}, x_{k}$ and $y_{k}$ of Eq. (10) using Gauss-Lobatto points.

\begin{tabular}{c|c|c|c}
\hline$k$ & $c_{k}$ & $x_{k}$ & $y_{k}$ \\
\hline \multicolumn{4}{c}{ Order of Gauss-Lobatto Quadrature rule, $\mathrm{s}=3$} \\
\hline 1 & 0.055555555555556 & 1.0000000000000000 & 1.000000000000000 \\
2 & 0.111111111111111 & 0.500000000000000 & 0.500000000000000 \\
3 & 0.000000000000000 & 0.000000000000000 & 0.000000000000000 \\
4 & 0.333333333333333 & 1.000000000000000 & 0.000000000000000 \\
5 & 0.8888888888888889 & 0.250000000000000 & 0.250000000000000 \\
6 & 0.1111111111111111 & 0.5000000000000000 & 0.500000000000000 \\
7 & 0.111111111111111 & 1.000000000000000 & 1.000000000000000 \\
8 & 0.333333333333333 & 0.000000000000000 & 1.000000000000000 \\
9 & 0.055555555555556 & 1.000000000000000 & 1.000000000000000 \\
\hline \multicolumn{4}{c}{ Order of Gauss-Lobatto Quadrature rule, $\mathrm{s}=4$} \\
\hline 1 & 0.0138888888888889 & 1.0000000000000000 & 1.0000000000000000 \\
2 & 0.050250472065971 & 0.723606797749979 & 0.723606797749979 \\
3 & 0.019193972378474 & 0.276393202250021 & 0.276393202250021 \\
4 & 0.000000000000000 & 0.000000000000000 & 0.000000000000000 \\
\hline
\end{tabular}




\begin{tabular}{|c|c|c|c|}
\hline & Table 2 (Continued) & & \\
\hline 5 & 0.088638416822918 & 1.000000000000000 & 0.447213595499958 \\
\hline 6 & 0.347222222222222 & 0.647213595499958 & 0.247213595499958 \\
\hline 7 & 0.191939723784737 & 0.076393202250021 & 0.076393202250021 \\
\hline 8 & 0.019193972378474 & 0.276393202250021 & 0.276393202250021 \\
\hline 9 & 0.119694916510415 & 1.000000000000000 & 0.447213595499958 \\
\hline 10 & 0.502504720659707 & 0.523606797749979 & 0.523606797749979 \\
\hline 11 & 0.347222222222222 & 0.247213595499958 & 0.647213595499958 \\
\hline 12 & 0.050250472065971 & 0.723606797749979 & 0.723606797749979 \\
\hline 13 & 0.027777777777778 & 1.000000000000000 & 1.000000000000000 \\
\hline 14 & 0.119694916510415 & 0.447213595499958 & 1.000000000000000 \\
\hline 15 & 0.088638416822918 & 0.447213595499958 & 1.000000000000000 \\
\hline 16 & 0.013888888888889 & 1.000000000000000 & 1.000000000000000 \\
\hline \multicolumn{4}{|c|}{ Order of Gauss-Lobatto Quadrature rule, s = 5} \\
\hline 1 & 0.005000000000000 & 1.000000000000000 & 1.000000000000000 \\
\hline 2 & 0.022521674962414 & 0.827326835353989 & 0.827326835353988 \\
\hline 3 & 0.017777777777778 & 0.500000000000000 & 0.500000000000000 \\
\hline 4 & 0.004700547259808 & 0.172673164646012 & 0.172673164646012 \\
\hline 5 & 0.000000000000000 & 0.000000000000000 & 0.000000000000000 \\
\hline 6 & 0.031922769482030 & 1.000000000000000 & 0.654653670707977 \\
\hline 7 & 0.148209876543210 & 0.797510813565120 & 0.511796527850834 \\
\hline 8 & 0.130216237304314 & 0.413663417676994 & 0.240990253030983 \\
\hline 9 & 0.051183736829021 & 0.029816021788869 & 0.029816021788869 \\
\hline 10 & 0.004700547259808 & 0.172673164646012 & 0.172673164646012 \\
\hline 11 & 0.053333333333333 & 1.000000000000000 & 0.000000000000000 \\
\hline 12 & 0.256944256522846 & 0.740990253030983 & 0.086336582323006 \\
\hline 13 & 0.252839506172840 & 0.250000000000000 & 0.250000000000000 \\
\hline 14 & 0.130216237304314 & 0.240990253030983 & 0.413663417676994 \\
\hline 15 & 0.017777777777778 & 0.500000000000000 & 0.500000000000000 \\
\hline 16 & 0.049743897184636 & 1.000000000000000 & 0.654653670707977 \\
\hline 17 & 0.245236016257398 & 0.684469692496846 & 0.684469692496846 \\
\hline 18 & 0.256944256522846 & 0.086336582323006 & 0.740990253030983 \\
\hline 19 & 0.148209876543210 & 0.511796527850834 & 0.797510813565120 \\
\hline 20 & 0.022521674962414 & 0.827326835353988 & 0.827326835353989 \\
\hline 21 & 0.010000000000000 & 1.000000000000000 & 1.000000000000000 \\
\hline 22 & 0.049743897184636 & 0.654653670707977 & 1.000000000000000 \\
\hline 23 & 0.053333333333333 & 0.000000000000000 & 1.000000000000000 \\
\hline 24 & 0.031922769482030 & 0.654653670707977 & 1.000000000000000 \\
\hline 25 & 0.005000000000000 & 1.000000000000000 & 1.000000000000000 \\
\hline
\end{tabular}

\section{Composite Integration over Standard Triangle ST}

Let us discretize $S T$ in $(u, v)$ space into $4(n \times n)=4 n^{2}$ right isosceles triangle $T_{i}$ each of area $1 /\left(2 n^{2}\right)$ [14]. This is depicted in Fig. 3. Let $F(u, v)=f(u, v)\left|J_{1}\right|$. Then 


$$
I=\iint_{A T} f(x, y) d y d x=\iint_{S T} f(u, v)\left|J_{1}\right| d u d v=\sum_{i=1}^{4(n \times n)} \iint_{T_{i}} F(u, v) d u d v
$$

Now each $T_{i}$ is to be transformed again into a standard triangle in $(X, Y)$ space, say. Observe that each $T_{i}$ is either Type I (Fig. 4a) whose vertices are $1\left(-1+\frac{i}{n},-1+\frac{j}{n}\right.$, $2\left(-1+\frac{i+1}{n},-1+\frac{j}{n}\right)$ and $3\left(-1+\frac{i}{n},-1+\frac{j+1}{n}\right)$, or Type II (Fig.4b) whose vertices are $1\left(-1+\frac{i+1}{n},-1+\frac{j+1}{n}\right)$, $2\left(-1+\frac{i}{n},-1+\frac{j+1}{n}\right)$ and $3\left(-1+\frac{i+1}{n},-1+\frac{j}{n}\right)$.

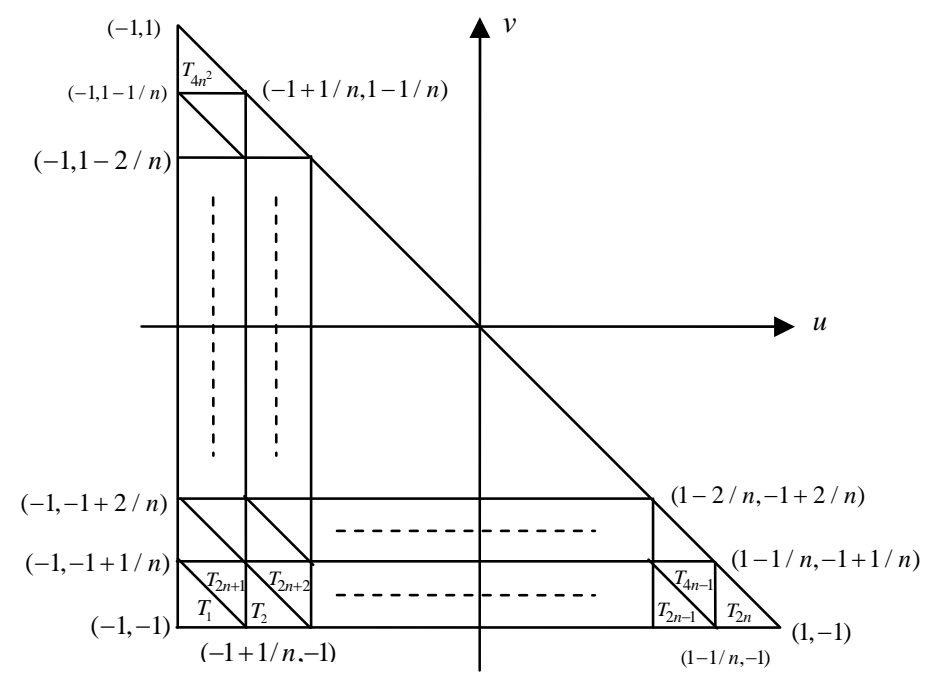

Fig. 3. Discretization of T into $4 n^{2}$ sub triangles $T_{i}$.

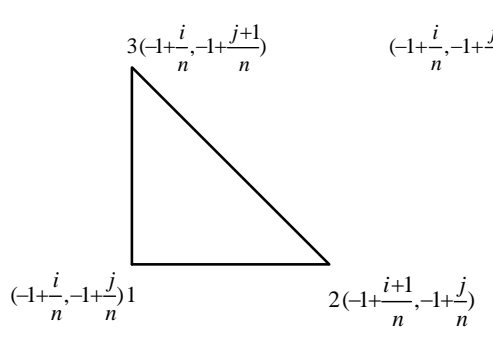

(a) Type-I

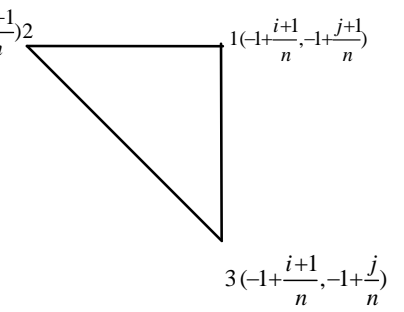

(b) Type-II

Fig. 4. Types of triangles of $T_{i}$ of Fig. 3 in $(u, v)$ space.

For type II triangles (Fig. 4b) 


$$
\begin{aligned}
u(X, Y) & =\left(-1+\frac{i+1}{n}\right)\left(-\frac{1}{2}(X+Y)\right)+\left(-1+\frac{i}{n}\right)\left(\frac{1}{2}(1+X)\right)+\left(-1+\frac{i+1}{n}\right)\left(\frac{1}{2}(1+Y)\right) \\
& =\frac{-X+2(i-n)+1}{2 n} \\
v(X, Y) & =\left(-1+\frac{j+1}{n}\right)\left(-\frac{1}{2}(X+Y)\right)+\left(-1+\frac{j+1}{n}\right)\left(\frac{1}{2}(1+X)\right)+\left(-1+\frac{j}{n}\right)\left(\frac{1}{2}(1+Y)\right) \\
& =\frac{-Y+2(j-n)+1}{2 n}
\end{aligned}
$$

Therefore from Eq. (11)

$$
I=\sum_{i=1}^{4(n \times n)} \iint_{T_{i}} F(u, v) d u d v=\frac{1}{4 n^{2}} \int_{-1}^{1} \int_{-1}^{-y} H(X, Y) d X d Y=\frac{1}{4 n^{2}} \int_{-1}^{1} \int_{-1}^{-X} H(X, Y) d Y d X
$$

where

$$
\begin{aligned}
H(X, Y)=\sum_{i=0}^{2 n-1} \sum_{j=0}^{2 n-1-i} F\left(\frac{X+2(i-n)+1}{2 n}, \frac{Y+2(j-n)+1}{2 n}\right) \\
+\sum_{i=0}^{2 n-2} \sum_{j=0}^{2 n-2-i} F\left(\frac{-X+2(i-n)+1}{2 n}, \frac{-Y+2(j-n)+1}{2 n}\right)
\end{aligned}
$$

We can now apply Gauss Legendre quadrature rules on the integral, in a manner similar to the procedure we already developed for integral $I=\iint_{A T} f(x, y) d y d x$ in previous section. Following the method already developed in previous section, we have now on using the transformation:

$$
X=\frac{1}{4}(-1+3 \xi-\eta(1+\xi)) \quad Y=\frac{1}{4}(-1+3 \eta-\xi(1+\eta))
$$

Therefore (12) becomes

$$
\begin{aligned}
I=\iint_{A T} f(x, y) d y d x & =\frac{1}{4 n^{2}} \iint_{S T} H(X, Y) d X d Y \\
= & \frac{1}{4 n^{2}} \int_{-1}^{1} \int_{-1}^{1} \frac{1}{4}(2-\xi-\eta) H(X(\xi, \eta), Y(\xi, \eta)) d \xi d \eta \\
& =\frac{1}{4 n^{2}} \sum_{p=1}^{s} \sum_{q=1}^{s} \frac{(2-\xi-\eta)}{8} W_{p} W_{q} H\left(X\left(\xi_{p}, \eta_{q}\right), Y\left(\xi_{p}, \eta_{q}\right)\right)
\end{aligned}
$$

where 


$$
\begin{aligned}
& H(X, Y)=\sum_{i=0}^{2 n-1} \sum_{j=0}^{2 n-1-i} F\left(\frac{X+2(i-n)+1}{2 n}, \frac{Y+2(j-n)+1}{2 n}\right) \\
& \quad+\sum_{i=0}^{2 n-2} \sum_{j=0}^{2 n-2-i} F\left(\frac{-X+2(i-n)+1}{2 n}, \frac{-Y+2(j-n)+1}{2 n}\right) \\
& X=X\left(\xi_{p}, \eta_{q}\right)=\frac{1}{4}\left(-1+3 \xi_{p}-\eta_{q}\left(1+\xi_{p}\right)\right) \\
& Y=Y\left(\xi_{p}, \eta_{q}\right)=\frac{1}{4}\left(-1+3 \eta_{q}-\xi_{p}\left(1+\eta_{q}\right)\right)(p, q=1,2,3, \ldots \ldots, s)
\end{aligned}
$$

From Eqs. (10)-(14), it is clear that we can obtain the following composite integration rule:

$$
I=\frac{1}{4 n^{2}} \sum_{k=1}^{N=s \times s} c_{k} H\left(x_{k}, y_{k}\right)
$$

where

$$
\begin{array}{r}
H\left(x_{k}, y_{k}\right)=\sum_{i=0}^{2 n-1} \sum_{j=0}^{2 n-1-i} F\left(\frac{x_{k}+2(i-n)+1}{2 n}, \frac{y_{k}+2(j-n)+1}{2 n}\right) \\
+\sum_{i=0}^{2 n-2} \sum_{j=0}^{2 n-2-i} F\left(\frac{-x_{k}+2(i-n)+1}{2 n}, \frac{-y_{k}+2(j-n)+1}{2 n}\right),
\end{array}
$$

and

$$
\begin{aligned}
& c_{k}=\frac{1}{4}\left(2-\xi_{p}-\eta_{q}\right) w_{p} w_{q} \\
& x_{k}=\frac{1}{4}\left(-1+3 \xi_{p}-\eta_{q}\left(1+\xi_{p}\right)\right) \quad(k=1,2, \ldots \ldots \ldots \ldots, N),(i, j=1,2, \ldots \ldots \ldots \ldots, s) \\
& y_{k}=\frac{1}{4}\left(-1+3 \eta_{q}-\xi_{p}\left(1+\eta_{q}\right)\right)
\end{aligned}
$$

\section{Numerical Examples}

In this section we estimate errors for three simple examples to show that the present formulation converges to exact solution with great accuracy than that of the conventional Gauss-Radau and Gauss-Lobatto quadratures.

$$
I_{1}=\iint_{R}(x+y)^{-1 / 2} d y d x=1.995191271069104,
$$

where $R$ is a triangular region connecting with the points $(-1,2),(2,1)$ and $(3,3)$. 


$$
\begin{aligned}
& I_{2}=\int_{0}^{1} \int_{0}^{x} e^{|x+y-1|} d y d x=0.718281834100861, \text { and } \\
& I_{3}=\int_{-1}^{1} \int_{-1}^{-x} \sin \left(x^{2}+y^{2}\right) d y d x=1.122580796643812 .
\end{aligned}
$$

In Table 3, errors of integrals are calculated by using Table 1, in which sampling points are generated by using Gauss-Radau points. In Table 4, errors of integrals are calculated by using Table 1, in which sampling points are generated by using GaussLobatto points. Finally in Table 5, errors of integrals are calculated by conventional Gauss-Radau and Gauss-Lobatto quadratures. The errors given in Table 3 and Table 4 are very negligible compared to those of Table 5.

\begin{tabular}{|c|c|c|c|}
\hline $4 \times n^{2}$ & $I_{1}$ & $I_{2}$ & $I_{3}$ \\
\hline \multicolumn{4}{|c|}{ Gauss-Radau Quadrature rule, s = 3} \\
\hline $4 \times 1^{2}$ & 0.00042 & 0.00423 & 0.00179 \\
\hline $4 \times 2^{2}$ & 0.00003 & 0.00106 & 0.00011 \\
\hline $4 \times 3^{2}$ & $5.05972 \times 10^{-6}$ & 0.00047 & 0.00002 \\
\hline $4 \times 4^{2}$ & $1.55175 \times 10^{-6}$ & 0.00027 & $6.46490 \times 10^{-6}$ \\
\hline $4 \times 5^{2}$ & $6.23037 \times 10^{-7}$ & 0.00017 & $2.64101 \times 10^{-6}$ \\
\hline $4 \times 6^{2}$ & $2.96591 \times 10^{-7}$ & 0.00012 & $1.27179 \times 10^{-6}$ \\
\hline $4 \times 7^{2}$ & $1.58702 \times 10^{-7}$ & 0.00009 & $6.85876 \times 10^{-7}$ \\
\hline $4 \times 8^{2}$ & $9.24637 \times 10^{-8}$ & 0.00007 & $4.01818 \times 10^{-7}$ \\
\hline $4 \times 9^{2}$ & $5.74720 \times 10^{-8}$ & 0.00005 & $2.50755 \times 10^{-7}$ \\
\hline $4 \times 10^{2}$ & $3.75849 \times 10^{-8}$ & 0.00004 & $1.64474 \times 10^{-7}$ \\
\hline \multicolumn{4}{|c|}{ Gauss-Radau Quadrature rule, s = 4} \\
\hline $4 \times 1^{2}$ & 0.00002 & 0.00228 & 0.00002 \\
\hline $4 \times 2^{2}$ & $4.84714 \times 10^{-7}$ & 0.00057 & $3.66263 \times 10^{-7}$ \\
\hline $4 \times 3^{2}$ & $4.56336 \times 10^{-8}$ & 0.00025 & $3.33264 \times 10^{-8}$ \\
\hline $4 \times 4^{2}$ & $8.18940 \times 10^{-9}$ & 0.00014 & $5.98998 \times 10^{-9}$ \\
\hline $4 \times 5^{2}$ & $2.13738 \times 10^{-9}$ & 0.00009 & $1.57668 \times 10^{-9}$ \\
\hline $4 \times 6^{2}$ & $7.11321 \times 10^{-10}$ & 0.00006 & $5.29143 \times 10^{-10}$ \\
\hline $4 \times 7^{2}$ & $2.80489 \times 10^{-10}$ & 0.00005 & $2.10100 \times 10^{-10}$ \\
\hline $4 \times 8^{2}$ & $1.25297 \times 10^{-10}$ & 0.00004 & $9.43656 \times 10^{-11}$ \\
\hline $4 \times 9^{2}$ & $6.15765 \times 10^{-11}$ & 0.00003 & $4.65725 \times 10^{-11}$ \\
\hline $4 \times 10^{2}$ & $3.26277 \times 10^{-11}$ & 0.00002 & $2.47597 \times 10^{-11}$ \\
\hline \multicolumn{4}{|c|}{ Gauss-Radau Quadrature rule, s = 5} \\
\hline $4 \times 1^{2}$ & $1.20974 \times 10^{-6}$ & 0.00143 & $3.82664 \times 10^{-7}$ \\
\hline $4 \times 2^{2}$ & $1.17070 \times 10^{-8}$ & 0.00036 & $8.78387 \times 10^{-10}$ \\
\hline $4 \times 3^{2}$ & $5.78610 \times 10^{-10}$ & 0.00016 & $2.77998 \times 10^{-11}$ \\
\hline $4 \times 4^{2}$ & $6.27607 \times 10^{-11}$ & 0.00009 & $2.55751 \times 10^{-12}$ \\
\hline $4 \times 5^{2}$ & $1.08593 \times 10^{-11}$ & 0.00006 & $4.12559 \times 10^{-13}$ \\
\hline $4 \times 6^{2}$ & $2.55840 \times 10^{-12}$ & 0.00004 & $9.48130 \times 10^{-14}$ \\
\hline $4 \times 7^{2}$ & $7.50733 \times 10^{-13}$ & 0.00003 & $2.79776 \times 10^{-14}$ \\
\hline $4 \times 8^{2}$ & $2.59792 \times 10^{-13}$ & 0.00002 & $9.99201 \times 10^{-15}$ \\
\hline $4 \times 9^{2}$ & $1.03251 \times 10^{-13}$ & 0.00002 & $4.66294 \times 10^{-15}$ \\
\hline $4 \times 10^{2}$ & $4.59632 \times 10^{-14}$ & 0.00001 & $2.66454 \times 10^{-15}$ \\
\hline
\end{tabular}

Table 3. Errors of integrals in which sampling points are generated by using Gauss-Radau points. 
The error is calculated as follows

Error $=\mid($ Exact value - Approximate value $) \mid$

Table 4. Errors of integrals in which sampling points are generated by using Gauss-Lobatto points.

\begin{tabular}{|c|c|c|c|}
\hline $4 \times n^{2}$ & $I_{1}$ & $I_{2}$ & $I_{3}$ \\
\hline \multicolumn{4}{|c|}{ Gauss-Lobatto Quadrature rule, s = 3} \\
\hline $4 \times 1^{2}$ & 0.00247 & 0.00686 & 0.01439 \\
\hline $4 \times 2^{2}$ & 0.00022 & 0.00173 & 0.00084 \\
\hline $4 \times 3^{2}$ & 0.00005 & 0.00077 & 0.00016 \\
\hline $4 \times 4^{2}$ & 0.00002 & 0.00043 & 0.00005 \\
\hline $4 \times 5^{2}$ & $6.67386 \times 10^{-6}$ & 0.00028 & 0.00002 \\
\hline $4 \times 6^{2}$ & $3.26412 \times 10^{-6}$ & 0.00019 & $9.84195 \times 10^{-6}$ \\
\hline $4 \times 7^{2}$ & $1.77757 \times 10^{-6}$ & 0.00014 & $5.30244 \times 10^{-6}$ \\
\hline $4 \times 8^{2}$ & $1.04816 \times 10^{-6}$ & 0.00011 & $3.10437 \times 10^{-6}$ \\
\hline $4 \times 9^{2}$ & $6.57073 \times 10^{-7}$ & 0.00009 & $1.93641 \times 10^{-6}$ \\
\hline $4 \times 10^{2}$ & $4.32402 \times 10^{-7}$ & 0.00007 & $1.26971 \times 10^{-6}$ \\
\hline \multicolumn{4}{|c|}{ Gauss-Lobatto Quadrature rule, $\mathrm{s}=4$} \\
\hline $4 \times 1^{2}$ & 0.00010 & 0.00316 & 0.00008 \\
\hline $4 \times 2^{2}$ & $3.37072 \times 10^{-6}$ & 0.00079 & $2.70476 \times 10^{-6}$ \\
\hline $4 \times 3^{2}$ & $3.89712 \times 10^{-7}$ & 0.00035 & $2.51565 \times 10^{-7}$ \\
\hline $4 \times 4^{2}$ & $7.87776 \times 10^{-8}$ & 0.00020 & $4.54702 \times 10^{-8}$ \\
\hline $4 \times 5^{2}$ & $2.21231 \times 10^{-8}$ & 0.00013 & $1.19959 \times 10^{-8}$ \\
\hline $4 \times 6^{2}$ & $7.72044 \times 10^{-9}$ & 0.00009 & $4.03050 \times 10^{-9}$ \\
\hline $4 \times 7^{2}$ & $3.14377 \times 10^{-9}$ & 0.00006 & $1.60140 \times 10^{-9}$ \\
\hline $4 \times 8^{2}$ & $1.43645 \times 10^{-9}$ & 0.00005 & $7.19568 \times 10^{-10}$ \\
\hline $4 \times 9^{2}$ & $7.17607 \times 10^{-10}$ & 0.00004 & $3.55230 \times 10^{-10}$ \\
\hline $4 \times 10^{2}$ & $3.84926 \times 10^{-10}$ & 0.00003 & $1.88892 \times 10^{-10}$ \\
\hline \multicolumn{4}{|c|}{ Gauss-Lobatto Quadrature rule, s = 5} \\
\hline $4 \times 1^{2}$ & $5.18813 \times 10^{-6}$ & 0.00182 & $3.22066 \times 10^{-6}$ \\
\hline $4 \times 2^{2}$ & $7.54983 \times 10^{-8}$ & 0.00046 & $7.36389 \times 10^{-9}$ \\
\hline $4 \times 3^{2}$ & $4.73682 \times 10^{-9}$ & 0.00020 & $2.27718 \times 10^{-10}$ \\
\hline $4 \times 4^{2}$ & $5.96499 \times 10^{-10}$ & 0.00011 & $2.06979 \times 10^{-11}$ \\
\hline $4 \times 5^{2}$ & $1.13768 \times 10^{-10}$ & 0.00007 & $3.30980 \times 10^{-12}$ \\
\hline $4 \times 6^{2}$ & $2.86215 \times 10^{-11}$ & 0.00005 & $7.48512 \times 10^{-13}$ \\
\hline $4 \times 7^{2}$ & $8.77809 \times 10^{-12}$ & 0.00004 & $2.13607 \times 10^{-13}$ \\
\hline $4 \times 8^{2}$ & $3.12350 \times 10^{-12}$ & 0.00003 & $7.17204 \times 10^{-14}$ \\
\hline $4 \times 9^{2}$ & $1.24745 \times 10^{-12}$ & 0.00002 & $2.68674 \times 10^{-14}$ \\
\hline $4 \times 10^{2}$ & $5.45786 \times 10^{-13}$ & 0.00002 & $1.11022 \times 10^{-14}$ \\
\hline
\end{tabular}

Table 5. Errors of integrals with conventional Gauss-Radau and Gauss-Lobatto quadratures.

\begin{tabular}{cccc}
\hline $\begin{array}{c}\text { Order of } \\
\text { quadrature }(s)\end{array}$ & $I_{1}$ & $I_{2}$ & $I_{3}$ \\
\hline \multicolumn{4}{c}{ Gauss-Radau Quadrature rule } \\
\hline 3 & 0.00521 & 0.01666 & 0.02830 \\
4 & 0.00050 & 0.00910 & 0.00026 \\
5 & 0.00006 & 0.00570 & 0.00007 \\
\hline 3 & Gauss-Lobatto Quadrature rule & \\
\hline 4 & 0.02165 & 0.02639 & 0.14217 \\
5 & 0.00169 & 0.01259 & 0.00598 \\
\end{tabular}




\section{Conclusion}

We have discussed, in details, the formulation of double integrals over an arbitrary triangular region. For this, we have transformed an arbitrary triangular region into a standard triangle $\{(u, v):-1 \leq u \leq 1,-1 \leq v \leq-u\}$ by the use of triangular basis functions. This standard triangle is then discretized into $4 \times n^{2}$ right isosceles triangles, in which the area of each of these triangles is $1 /\left(2 n^{2}\right)$. We then compute new $n^{2}$ sampling points and coefficients through the affine transformations via 2-sqaure, $\{(\xi, \eta):-1 \leq \xi, \eta \leq 1\}$ finite element by using the $n$-points Gauss-Radau, and Gauss-Lobatto quadratures. Numerical errors show that the present formulation converges to the exact solutions with a large significant digits compare to the conventional Gauss Radau and Gauss Lobatto quadratures.

\section{References}

1. P. C. Hammer, O. J. Marlowe and A. H. Stroud, Math. Tables and other aids to computation 10, 130 (1956).

2. P. C. Hammer and A. H. Stroud, Math. Tables and other aids to computation 12, 272 (1958).

3. A.H. Stroud, Numerical Quadrature and Solution of Ordinary Differential Equations, (Springer-Verlag, New York Berlin Heidelberg, 1974).

4. O. C. Zienkiewicz, The Finite Element Method, 3rd Ed. (Mc Graw Hill Inc., 1977).

5. G. R. Cowper, Inter. Jn. Numer. Methods Engrs. 7, 405, (1973).

6. J. N. Lyness and D. Jespersen, J. Inst. Math. Appl. 15, 19 (1975).

7. F. G. Lethor, J. Computational and Applied Mathematics 2, 219 (1976).

8. P. Hillion, Inter. Jn. Numer. Methods Engrs. 11, 797 (1977). doi:10.1002/nme.1620110504

9. F. G. Lannoy, Comput. Struct. 7, 613 (1977).

10. G. Lague and R. Baldur, Inter. Jn. Numer. Methods Engrs 11, 388 (1977). doi:10.1002/nme.1620110214

11. M. E. Lauresn and M. Gellert, Inter. Jn. Numer. Methods Engrs 12, 67 (1978). doi:10.1002/nme.1620120107

12. H.T. Rathod, K.V. Nagaraja and B. Venkatesudu, Appl. Math. Comput. 190, 21 (2007). doi:10.1016/j.amc.2009.01.030

13. M. S. Islam and G. Saha, Bangladesh J. Sci. Ind. Res. 43 (3), 377 (2008). doi:10.3329/bjsir.v43i3.1153

14. M. S. Islam and M. Alamgir Hossain, Appl. Math. Comput. 210 (2), 515 (2009). doi:10.1016/j.amc.2009.01.030 